\title{
O Uso do Tratamento Restaurador Atraumático na Saúde Pública
}

\author{
The Use of Atraumatic Restorative Treatment in Public Health
}

Recebido: 02/10/2021 | Revisado: 10/10/2021 | Aceito: 14/10/2021 | Publicado: 19/10/2021

\author{
Ariane Marcondes da Cruz Souza \\ Universidade Brasil, Brasil \\ E-mail: arianemarcondes1@gmail.com \\ Gabriellen Santana Capella \\ Universidade Brasil, Brasil \\ E-mail: gabriellen.capella@gmail.com \\ Jéssica Rayanne Mangueira de Pádua e Silva \\ Universidade Brasil, Brasil \\ E-mail: jessicapadua08@gmail.com \\ José Lucas Martins \\ Universidade Brasil, Brasil \\ E-mail: José.lucas@universidadebrasil.edu.br
}

\begin{abstract}
Resumo
O TRA- Tratamento Restaurador Atraumático revela-se como uma estratégia de controle da cárie dentária pelo sistema público de saúde, visto que a técnica apresenta baixo custo benefício e boa aceitação pelas comunidades da rede pública de saúde. Este método é minimamente invasivo, e consiste na remoção do tecido cariado, utilizando o cimento de ionômero de vidro como material restaurador, pois o mesmo tem a capacidade de remineralizar o esmalte dentário, promover a biocompatibilidade e reparar a dentina afetada. Trata-se de um procedimento bem visto pelos pacientes por descartar o uso de anestesia, uma vez que para a manipulação da técnica não é necessário todo o aparato odontológico avançado, já que seu manejo é simples e apresenta baixo custo operacional, adequando-se as dificuldades presentes no sistema público de saúde brasileiro. Este trabalho constitui no método de análise quantitativa descritiva e tem por finalidade mostrar o TRA como auxilio de tratamento da cárie dentária na rede pública de saúde.
\end{abstract}

Palavras-chave: TRA; Saúde pública; Cárie dentária.

\begin{abstract}
The ART - Atraumatic Restorative Treatment is a strategy of dental caries control by the public health system, considering that this technique demonstrates low cost-benefit and good acceptance by communities in the public health network. This method is minimally invasive, and consists of removing carious tissue, using glass ionomer cement as a restorative material, as it has the capacity to remineralize tooth enamel, to promote biocompatibility and repair the affected dentine. It is a procedure well regarded by patients as it discards the use of anesthesia, whereas all the technical handling does not need advanced dental apparatus, considering that is a simple handling with low operating cost, adapting to the difficulties present in brazilian public health system. This work constitutes the method of descriptive quantitative analysis and aims to show the ART as an aid in the treatment of dental caries in the public health network.
\end{abstract}

Keywords: ART; Public health; Dental cavity.

\section{Introdução}

Classificada com uma doença infecciosa que avança de forma vagarosa e afeta a saúde bucal dos indivíduos, a cárie dentária progride até causar lesões na estrutura do dente (Fejerskov et al. 2005).

Sabe-se que a cárie é uma doença causada pelo microrganismo Streptococcus mutans que ao se metabolizar com carboidratos fermentáveis como, por exemplo, a sacarose, produz ácidos que corroem a coroa do dente, desmineralizando o esmalte dentário e formando lesões (Stephan, 1940, Gustafsson et al. 1954).

Estas alterações da estrutura dentária têm como fatores determinantes a dieta rica em carboidratos, biofilme e o tempo de interação entre o hospedeiro e o indivíduo, que por sua vez determina a desmineralização; além disso, fatores sociais, 
comportamentais e econômicos também influenciam no surgimento e desenvolvimento da doença. (Antunes et al. 2004, Keyes, 1960). Entender a epidemiologia da doença é importante para definir e estabelecer o programa de prevenção e tratamento, além de designar um planejamento odontológico adequado.

Mesmo com a redução da doença em países desenvolvidos, o problema ainda atinge uma grande porcentagem das pessoas de todas as comunidades segundo a Organização Mundial da Saúde (OMS), pois em países subdesenvolvidos este índice não diminuiu significativamente e o tratamento da cárie ainda não é executado devido á falta de recursos financeiros por parte dos pacientes, que acabam optando pela extração dentária ao invés do tratamento. (Prado. 2008).

O TRA-Tratamento Restaurador Atraumático ou sua terminologia no inglês ART, (Atraumatic Restorative Treatment) é uma metodologia que trata cárie a dentária, sendo um procedimento minimamente invasivo que constitui basicamente em escavar a dentina infectada com instrumentais manuais odontológicos e logo após, uma restauração definitiva da cavidade ou fissuras com material adesivo restaurador. Uma das vantagens é que se trata de uma técnica conservadora, uma vez que só remove o tecido cariado e descarta a aplicação de anestesia, e possui boa aceitação tanto para os pacientes quanto para os cirurgiões dentistas da rede pública de saúde, uma vez que este método não necessita ser realizado exclusivamente no consultório odontológico, pois seu manuseio é simples e apresenta baixo custo operacional. (Frencken et al. 2001).

Além disso, para tal técnica recomenda-se o uso do cimento de ionômero de vidro como material restaurador, que possui boa adesão química ao esmalte e a dentina: que entre suas propriedades está à liberação do flúor que promove a biocompatibilidade com o tecido pulpar e sua remineralizarão inibe o número de bactérias e o seu metabolismo, sela a cavidade, repara a dentina afetada e favorece resposta do complexo dentino-pulpar.

O TRA é eficaz no tratamento da cárie, principalmente em comunidades mais carentes, que utilizam da rede pública de saúde, e é aplicado na realidade e na rotina do cirurgião dentista, pois um dos problemas enfrentados por esses profissionais da saúde pública é a escassez de equipamentos adequados para o tratamento restaurador.

Essa metodologia está associada á promoção da saúde ofertada pela rede de saúde pública, o SUS (Sistema Único de Saúde), criado em 1988 pela Constituição Federal Brasileira, mas foi apenas em 2004 que se deu início a maior política de saúde bucal do mundo, o Programa Nacional de Saúde Bucal - Programa Brasil Sorridente, que em 2020 completou 16 anos de atuação. (Ministério da Saúde, 2018. 350. Pag 11).

Atualmente, o Brasil tem o menor índice de prevalência de cárie comparado com a média dos países da América Latina, segundo dados da OMS, porém, o país ainda sofre com a falta de recursos e materiais para atender a demanda da população brasileira. A Ação Primária a Saúde - APS é um nível primário que tem como função resolver os problemas mais frequentes de saúde da população, com o propósito de minimizar os custos econômicos e atender a demanda dos brasileiros. (Brasil, 2015).

O objetivo desse trabalho é apresentar a aplicação do TRA no sistema público de saúde como forma tratamento no auxílio do controle de cárie dentária pelos dentistas que atuam na rede pública e expor seus benefícios.

\section{Metodologia}

Este estudo utiliza como método a análise quantitativa descritiva, a partir de uma bibliografia que têm como objetivo entender o conhecimento do TRA - Tratamento Restaurador Atraumático na saúde pública como ação primária na atenção básica a saúde e o conhecimento prático do cirurgião dentista através da técnica, sendo o TRA o mais eficaz em frente a ausência de aparatos odontológicos avançados na rede pública brasileira. Para a realização deste artigo, foram utilizados estudos bibliográficos. Estrela, C. (2018). 


\section{Revisão de Literatura}

\section{Cárie dentária}

A cárie é uma doença multifatorial infectocontagiosa, que ocorre a partir de uma dieta rica em carboidratos, condições socioeconômicas precárias e má higiene bucal do indivíduo; acometendo de forma crônica e agressiva o elemento dentário, levando até a sua perda total. O fator etiológico da cárie é a colonização de microrganismos aderidos à superfície do esmalte, sendo eles o Streptococcus mutans um dos principais agentes, quando em contato com carboidratos fermentáveis geram ácidos que desmineraliza o esmalte dentário. (Pettersen, 2003, Pitts et al., 2017).

Visto que o meio bucal é acometido por uma diversidade de doenças, a que têm maior prevalência é a cárie dentária. Segundo Pettersen (2003), relata que a cárie é classificada como principal problema de saúde pública em países em desenvolvimento, afetando cerca de 60 a $90 \%$ de crianças no período escolar.

Sendo assim, a odontologia apresenta métodos e/ou técnicas de tratamentos para este tipo de lesão.

\section{Tratamento restaurador atraumático}

Em 1980, Frencken recomendou uma técnica alternativa para o tratamento do controle da cárie dentária, um tratamento pouco invasivo e com procedimentos simples, podendo ser realizado em qualquer lugar sem a necessidade de grandes recursos para efetuar o procedimento. (Gomes, 1999, Gomes, et al. 2003, Nunes et al. 2003)

Contudo, esta metodologia foi pronunciada em 7 de Abril de 1994 para a OMS e à comunidade cientifica. Desde então, se dissipou rapidamente em diversos países. Os primeiros testes da técnica tiveram inicio na Universidade de Groninen Holanda (Manssoni et al. 2006).

Essa técnica criada por Frencken tinha o intuito de acolher pacientes que residiam em regiões carentes no continente africano e que enfrentavam dificuldades monetárias e falta de energia elétrica, impossibilitando a realização de Tratamentos Odontológicos convencionais. Este procedimento foi nomeado de Tratamento Restaurador Atraumático, que vêm do inglês “Atraumatic Restorative Treatement” (ART) e visava reduzir o incomodo das lesões causadas pela cárie gerando á perda do elemento dentário decorrente á progressão cariosa. (Wambier et al. 2003).

A metodologia aplicada remove o tecido cariado, amolecido e infectado, através de instrumentais de uso manual, e para preenchimento da cavidade dentária utiliza materiais com a função de suspender a progressão da cárie e diminuir o surgimento de novas lesões. (Araújo et al. 2000).

A utilização de materiais manuais torna essa técnica vantajosa, já que não é necessária a aplicação de anestesia, outro benefício dessa técnica é a locomoção desses materiais entre os ambientes. A TRA busca preservar a dentina afetada com o intuito de ser passível de remineralização. A técnica isenta o uso de equipamentos odontológicos tradicionais e de rotação que necessitam de energia elétrica para funcionar. (Figueiredo et al. 2004).

O material de eleição do TRA é o Cimento de Ionômero de Vidro (CIV), pois ele tem como propriedade liberação de íons de flúor que têm a capacidade de remineralizar o tecido dentinário remanescente. (Araújo et al. 2017, Asakawa et al. 2017)

Outras propriedades do CIV é a biocompatibilidade, a adesão química, além do fator de expansão térmica semelhante às estruturas dentais (Nicholson et al, 2020). De acordo com Guideline on Pediatric Restorative Dentistry, 2006, o CIV apresenta várias características que são favoráveis ao uso em crianças.

Para a abertura da cavidade contamos com o auxílio de instrumentais manuais que tem por finalidade facilitar o acesso á cavidade. 
Segundo Medonça (2009), os materiais utilizados são:

- Machado (de forma piramidal);

- Curetas de diferentes tamanhos;

- Espátula de inserção;

- Esculpidor;

- Espátula para manipulação do CIV;

- Pinça clinica;

- Espelho bucal;

- Sonda exploradora;

- CIV;

- Bolinhas e rolete de algodão;

- Vaselina;

- Verniz ou esmalte incolor;

- $\quad$ Placa de vidro;

- Potes para água;

- Matriz metálica;

- Cunha de madeira;

- $\quad$ Papel Carbono (checar oclusão).

É também necessária à utilização de EPIs, de acordo com Mjör (1999), são eles:

- Touca;

- Máscara;

- Luvas;

- $\quad$ Óculos de proteção.

Análise de tabela sobre as vantagens e desvantagens do TRA.

Tabela 1 - Vantagens e Desvantagens do TRA.

\begin{tabular}{|c|c|}
\hline Vantagens & Desvantagens \\
\hline $\begin{array}{l}\text { Fácil utilização dos materiais, não sendo necessários } \\
\text { equipamentos de uso elétrico; }\end{array}$ & Não é indicada para todas as classes de restauração; \\
\hline Pode ser realizada em qualquer local; & $\begin{array}{l}\text { Uma técnica que nem todo cirurgião domina pela falta de } \\
\text { conhecimento, ocasionando fracasso nas restaurações; }\end{array}$ \\
\hline $\begin{array}{l}\text { Não é necessário fazer o isolamento absoluto e nem } \\
\text { anestesiar; }\end{array}$ & $\begin{array}{l}\text { Exaustão do profissional pelo fato de utilizar somente } \\
\text { instrumentais manuais; }\end{array}$ \\
\hline Garante conforto ao paciente durante o atendimento; & $\begin{array}{l}\text { Problemas decorrentes da sinérese e embebição pela } \\
\text { propriedade mecânica do CIV. }\end{array}$ \\
\hline \multicolumn{2}{|l|}{$\begin{array}{l}\text { Pode ser aplicado em crianças, adultos, gestante, idosos, } \\
\text { portadores de necessidades especiais e com } \\
\text { comprometimento imunológico; }\end{array}$} \\
\hline Baixo custo benefício; & \\
\hline Aplicada á pessoas em situações carentes. & \\
\hline
\end{tabular}

Fonte: Mendonça C.S. (2009), Massoni A.C.L.T. et al. (2006). 
De acordo com a Tabela 1, foram observadas vantagens e desvantagens no TRA, as quais podem observar que é uma técnica facilitadora e confortável para sua realização.

\section{TRA e sua utilização em saúde pública}

A técnica do TRA não necessariamente deve ser considerada uma alternativa de tratamento, mas deve ser visualizada como uma estratégia de promoção de saúde bucal, englobando a população menos favorecida, pois se adéqua a realidade da mesma. Sendo assim, ela incorpora tais ações como: curativas, promoção e prevenção de saúde. (Frencken et al. 1999).

Sendo um procedimento indicado ao controle de cárie em lugares com baixa infraestrutura, a utilização do TRA é pouco absorvida entre os programas de saúde pública. (Massoni et al. 2006).

Contudo, a OMS solicitou a utilização da técnica em conjunto às atividades preventivas nos programas de promoção de saúde (Frencken et al. 1994). Segundo Figueiredo et al (2004) foi proposto as escolas públicas uma ação curativa através do tratamento restaurador atraumático para todas as crianças, tendo como objetivo reduzir a doença atual e possível aparecimento de novos casos.

Na odontologia foi inserido um programa denominado Estratégia da Saúde da Família, ESF, na edição da Portaria 1.444, de 28/12/2000, com o intuito de reorganizar á atenção á saúde bucal. Seu objetivo principal é a ampliação de acesso às ações integrais, através de medidas de caráter coletivo pelas equipes de trabalho em saúde bucal, substituindo as práticas tradicionais. (Kuhnen et al. 2013)

Os índices de saúde bucal nos serviços públicos em diversos países sofrem impasses de se estabelecerem com bons resultados, tendo como uma opção de tratamento propicia o TRA, possibilitando se tornar viável á toda população, tanto da classe baixa como a da alta. A atenção primaria no Brasil cresce de forma progressiva e sua técnica é considerada útil e apta de ser empregada na inclusão social do SUS. (Mendonça 2009)

Diversos estudos foram realizados ao longo dos anos, visando à atribuição da TRA para controle da lesão cariosa no sistema público de saúde. Diante disso a técnica apresenta um alto índice de sucesso nas restaurações, além disso, traz conforto ao paciente durante ao atendimento, pois é considerado indolor, também diminui a ansiedade, o estresse e o tempo de trabalho. (Fonseca et al. 2010).

Para o Programa de Saúde da Família, PSF, o TRA apresenta uma ação favorável, adequando-se aos indicadores socioeconômicos de saúde bucal da população. Para o PSF todo cidadão é o principal responsável pela busca de uma melhora em sua qualidade de vida, independentemente de sua realidade social, e para isto o mesmo deve ser tratado de maneira contextualizada. (Mossani et al. 2006).

\section{Discussão}

A técnica restauradora traumática foi elaborada pela necessidade de combater as cáries que provocavam sérios danos aos pacientes, geralmente resultando na perda dos componentes dentários e desenvolvida originalmente para atender pessoas que não podem contar com equipamentos odontológicos qualificados. Porém, devido aos excelentes resultados, passou a ser utilizado principalmente por profissionais da rede pública de saúde (Marra et al. 2008).

Apesar de que Frencken e Holmgren (1999) indiquem o uso dessa tecnologia para combater a cárie dentária em pessoas com baixo nível socioeconômico, Phantumvanit et al. 1996 acreditam que essa tecnologia é aplicável a toda a população.

Além do mais, a técnica TRA não deve ser apenas considerada uma tecnologia de reparo alternativo, mas também deve ser avaliada dentro da sua área de aplicação, a complexidade de uma estratégia de promoção de saúde bucal visa 
proporcionar tratamento odontológico para as populações não vistas. Assim sendo, esse tratamento articula ações de educação e saúde preventiva com a população, fases de prevenção, ações de tratamento para retirada de tecido cariado com instrumentos manuais e preparo cavitário. (Frencken et al. 1999).

Opiniões diferentes sobre os conceitos filosóficos da técnica são contraditórias e expuseram suspeitas entre os operadores, porque embora a maioria dos dentistas saiba que a TRA é eficaz, menos de um terço desses profissionais não utilizaram seu tempo clínico para serviços público. Já os princípios técnicos da TRA opiniões se dividem quando metades dos operadores discordam que é mais fácil utilizar as brocas para executar restaurações, enquanto a outra metade prefere utilizar essa técnica por achar que é "mais limpo". (Carlotto et al. 2013)

Contudo sobre TRA, as evidências científicas atuais mostram que a tecnologia é uma alternativa possível para o tratamento de cárie dentária no setor público de saúde, ajudando a amenizar problemas relacionados à grande demanda por tratamento suprimido. (Lima et al. 2008)

\section{Considerações Finais}

Diante deste estudo, apesar da abordagem do SUS que utiliza fluoretação no abastecimento de água e promoção a saúde bucal como medida de prevenção da cárie dentária, mesmo assim não foi possível impedir o desenvolvimento da doença, que infelizmente ainda prevalece na população brasileira.

Com base nisso o Sistema Único de Saúde - SUS utilizou a técnica do TRA uma das mais acessíveis originalmente criada para pessoas carentes, podendo ser utilizada para qualquer classe socioeconômica como método de minimizar os danos causados pela cárie. Apesar disso o TRA ainda é pouco utilizado pelos cirurgiões dentista devido à falta de conhecimento e a manipulação da técnica.

É importante certificar que o profissional odontólogo deve ter o conhecimento prático das técnicas, para alcançar resultados satisfatórios e apresentar eficácia no tratamento, ele deve compreender as recomendações e as limitações presentes na rede pública como também a de seus pacientes que utiliza o sistema público de saúde.

Pode-se concluir que o cirurgião dentista deve ter conhecimento da técnica empregada com precisão, avaliando as indicações e as barreiras presentes no cotidiano e buscar a ser apto no assunto, além de colaborar para o paciente e a sociedade cientifica.

\section{Referências}

American Academy of Pediatric Dentistry Clinical Affairs Committee--Restorative Dentistry Subcommittee; American Academy of Pediatric Dentistry Council on Clinical Affairs. Guideline on pediatric restorative dentistry. Pediatr Dent. (7 Suppl):122-9.

Antunes, J. L. F., Narvai, P. C. \& Nugent, Z. J. (2004). Measuring inequalities in the distribution of dental caries. Community Dent Oral Epidemiol, 32(1), 4148 .

Araújo, J. F., Valois, E. M., Lago, A. D. N., Silva, B. M. A. H., Costa, J. F. \& Firoozmand, L. M. (2017). Remoção parcial do tecido cariado em dentes permanentes: uma revisão de literatura. Rev. Bras. Odontol. 74(1), 31-35. 10.18363/rbo.v74n1.p.31.

Asakawa, L. \& Franzin, L. C. S. (2017). Tratamento Restaurador Atraumático (ART): Uma visão contemporânea. Revista UNINGÁ. $29(1), 159-62$.

Estrela, C. (2018). Metodologia Científica: Ciência, Ensino, Pesquisa. Editora Artes Médicas.

Fejerskov, O. \& Kidd, E. (2005) Cárie dentária: a doença e seu tratamento clínico. Santos.

Figueiredo, C. H., Lima, F. A. \& Moura, K. S. (2004). Tratamento restaurador atraumático: avaliação de sua viabilidade como estratégia de controle da cárie dentária na saúde pública. RBPS, 17(3), 109-118.

Frencken, J. E., Phantumvanit, P., Hantumvanit, P. \& Pilot, T. (1994). Atraumatic Restor $\neg$ ative Treatment technique of dental caries. Groningen: WHO Collaborating centre for Oral Health Services Research, University of Groningen.

Frencken J.E. \& Holmgren C.J. (1999). How effective is ART in the management of caries? Community Dent Oral Epidemiol; 27:423-30. 
Fonseca, P. M. L., Oliveira, M. B. L. A. \& Domingos, S. A. P. (2010). Tratamento restaurador atraumático: alternativa viável para a promoção da saúde bucal. Uningá Review, Maringá. 3, 39- 49

Gomes, A. C., Biella V. A., Mastrantonio, S. D. S. \& Neves L. T. D. (2003). O tratamento restaurador atraumático (TRA) como alternativa de tratamento em bebês portadores de fissura: relato de caso clínico. Revista Odontológica de Araçatuba, 24(2), 52-55.

Gomes, J. C. (1999). Tratamento restaurador atraumático visando à promoção de saúde. 52 f. Monografia (Especialização) _ Faculdade de Ciências da Saúde, Universidade Vale do Rio Doce, Governador Valadares.

Gustafsson, B. E., Quensel, C. E. L., Lanke, L.S., Ludqvist, C., Grahnén, H., Bonow, B. E. \& Krasse, B. (1954). The effect of different levels of carbohydrate intake on caries activity in 436 individuals observed for five years. Acta Odontologica Scandinava, 11: 232-364.

Kuhnen, M., Buratto, G. \& Silva, M. P. (2013). The use of atraumatic restorative treatment in the Family Health Strategy. Rev Odontol UNESP. 42(4): 291297.

Lima, D.C., Saliba, N.A. \& Moimazm, S. A. S. (2008). Tratamento restaurador atraumático e sua utilização em saúde pública. RGO, 56(1), 75-79.

Massoni, A. C. L. T., Pessoa, C. P. \& Oliveira, A. F. B. (2006). Atraumatic restorative treatment and its application in public health. Rev Odontol da UNESP; 35 (3): 201-207.

Mendonça, C. S. (2009). Saúde da família, agora mais do que nunca. Ciênc. Saúde Colet.; 14(Supl. 1): $1493-7$.

Mjör, I. A. \& Gordan, V. V. (1999). A review of atraumatic restorative treatment (ART). IntDent J., 49(3), $127-31$.

Monnerat, A. F., Souza, M. I. C. \& Monnerat, A. B. L. (2013). Atraumatic Restorative Treatment. Can we trust in this technique? Rev. bras. odontol., 70(1), $33-6$.

Navarro, M. F. L., Leal, S. C., Molina, G. F, Villena, R. S. (2015). Tratamento Restaurador Atraumático: atualidades e perspectivas. Rev assoc paul cir dent. 69(3):289-301.

Nicholson, J. W., Sidhu, S. K., \& Czarnecka, B. (2020). Enhancing the mechanical properties of glass-ionomer dental cements: a review. Materials, 13(11), 2510 .

Nunes, O. B. C., Abreu, P. H., Nunes, N. A., Reis, L. P. K. F. M., Reis, R. T. M. \& Roberto Júnior, A. (2003). Avaliação clínica do tratamento restaurador atraumático (art) em crianças assentadas do movimento sem-terra. Rev. Fac. Odontol. 15 (1): 23-31.

Oliveira, L., Neves, A., Neves, M. \& Souza I. (1998). Tratamento restaurador atraumático e adequação do meio bucal. Rev. Bras Odontol; 55(2): 94-9.

Petersen, P. E. (2003). The World oral health report 2003: continuos improvement of oral health in the 21st century- the approach of the WHO Global Oral Health Programme. Community Dent Oral Epidemiol; 31 (Suppl. 1):3-23.

Pitts N.B., Zero D.T., Marsh P.D., Ekstrand K., Weintraub J.A. \& Gomez F. R. (2017). Dental Caries. Nature Reviews Disease Primers, 25(3), 17030.

Prado, M. P. M. (2008). ART - tratamento restaurador atraumático: uma opção de prevenção em odontologia de baixo custo e ampla cobertura. 22 f. Trabalho de conclusão de curso (Especialização) _ Escola de Saúde do Exército, Rio de Janeiro.

Stephan, R. M. (1940). Changes in hydrogenion concentration on tooth surfaces and in caries lesions. Journal of The American Dental Association. 27:718723.

Smales, R. J. (2000). The atraumatic restorative treatment (ART) approach for primary teeth: review of literature. The atraumatic restorative treatment (ART) approach for primary teeth: review of literature; 22(4):294-298.

Wambier, D. S., Paganini, F. \& Locatelli, F. A. (2003). Tratamento restaurador atraumático (estudo da sua aplicabilidade em escolares de Tangará - SC). Pesq Bras Odontoped Clín Integr, 3(2), 09-13.

https://www.gov.br/pt-br/noticias/saude-e-vigilancia-sanitaria/2020/01/pesquisa-ira-atualizar-a-situacao-da-saude-bucal-dos-brasileiros Pesquisa irá atualizar a situação da saúde bucal dos brasileiros.

Brasil. Ministério da Saúde. Secretaria de Atenção à Saúde. Departamento de Atenção Básica. A saúde bucal no Sistema Único de Saúde [recurso eletrônico] / Ministério da Saúde, Secretaria de Atenção à Saúde, Departamento de Atenção Básica. - Brasília: Ministério da Saúde, 2018. 350 p.: il. Modo de acesso: World Wide Web Saúde bucal. 2. Políticas públicas. 3. Sistema Único de Saúde (SUS). I.) PAG 11. 